\title{
Das Linguistische Antiquariat
}

Utz Maas: Grundkurs Sprachwissenschaft. Teil I: Die herrschende Lehre. München: List Verlag, 1973. 300 S. [2. erweiterte und verbesserte Auflage 1974, 3. Auflage mit verändertem Untertitel: „Teil I: Grammatiktheorie (Die herrschende Lehre)“, Frankfurt: Syndikat 1979.]

Besprochen von Ralf Klabunde: Bochum, E-Mail: klabunde@linguistics.rub.de https://doi.org/10.1515/zfs-2018-0010

1973 erschien das mit Passion und Gelehrsamkeit geschriebene Lehrbuch von Utz Maas Grundkurs Sprachwissenschaft mit dem Untertitel Teil I: Die herrschende Lehre. Angelegt war dieses Buch als Eröffnung eines dreibändigen Werks zur Linguistik, wobei die zwei ausstehenden Bände in der Vorbemerkung der 1. Auflage als Werke zu formalen Theorien außerhalb der „herrschenden Lehre“, insbesondere zur philosophischen Logik und zur Generativen Semantik angekündigt werden, sowie als ein auf der Kritik in den beiden vorherigen Bänden aufbauendes Werk zu einer Linguistik für den Ausbildungsbereich. Diese beiden Bände wurden meines Wissens nicht mehr realisiert, aber der erste Band erzielte offensichtlich eine hohe Resonanz, denn es folgten eine zweite Auflage 1974 sowie eine in ihrer anfänglichen Polemik entschärfte dritte Auflage 1979 mit entsprechend geändertem Untertitel - dort heißt es nur noch Teil 1: Grammatiktheorie. Gedacht war das Buch als Arbeitsgrundlage für Lehrveranstaltungen.

Ziel des ersten Bandes sei, „die herrschende Wissenschaft aus den Bedingungen für ihre Entwicklung heraus zu erklären, um so eine Kritik ihrer Einbeziehung in den Ausbildungssektor zu ermöglichen“ (S. 17). Dementsprechend charakterisieren die meisten Kapitel des Buchs die Entwicklung der Linguistik. Diese Kapitelfolge beginnt mit kursorischen Bemerkungen $\mathrm{zu}$ linguistischen Analysen und deren zugrunde liegenden gesellschaftlichen und politischen Bedingungen von der Antike bis zur Neuzeit und steigt dann detailliert bei der historisch-vergleichenden Sprachwissenschaft ein. Sie endet mit einer Auseinandersetzung mit dem vorherrschenden Paradigma in der Linguistik der 1970er Jahre, der von Chomsky propagierten Generativen Grammatik. Das Buch ist aber viel mehr als ein historischer Abriss der neueren Geschichte der Linguistik; Utz Maas spricht zu Beginn und am Ende des Buchs Fragen zum Gegenstandsbereich der Linguistik an, die in der heutigen Betrachtung zum Teil erstaunlich aktuell sind und zum Nachdenken über die Beziehung zwischen Linguistik und Gesellschaft anregen.

Aber der Reihe nach. Um das Buch angemessen würdigen zu können, muss man sich den politischen und gesellschaftlichen Hintergrund bewusst machen, 
der dem Bedürfnis des Autors, dieses Buch schreiben zu müssen, zugrunde liegt. Utz Maas lehrte längere Zeit in Westberlin. Folgt man den Erläuterungen in Ballmer (1985), müssen die 1960er und der Anfang der 1970er Jahre in Berlin für die dortigen Linguisten eine intellektuell äußerst produktive Zeit gewesen sein, in der eine Aufbruchstimmung hin zu einer modernen, international anschlussfähigen Linguistik herrschte - ironischerweise mitinitiiert von der Akademie der Wissenschaften in Ostberlin. Diese Ausrichtung erfolgte zu der Zeit der großen bundesdeutschen Bildungsreform der 1960er und 1970er Jahre, die unter anderem zu einem Boom der Linguistik führte. Dies zeigte sich z. B. in Überlegungen, Linguistik als Schulfach einzurichten, um über dieses Fach gesellschaftliche Hürden, die durch Sprachbarrieren begründet sind, abzubauen - unter der häufiger anzutreffenden Vermutung, Korrelation drücke Kausalität aus, zeigt sich hier wohl der Einfluss von Basil Bernsteins soziolinguistischer Arbeit auf die Bildungspolitik.

Mit diesen Überlegungen einhergehend spezialisierte sich die Linguistik aber zunehmend in diverse „Bindestrich-Linguistiken“, und insbesondere nahm die Generative Grammatik in der Linguistik eine dominante Stelle ein. Damit entstand eine Lücke zwischen einer Forschungsrichtung, die von jeglichen Aspekten der Kommunikationssituation abstrahiert, und einer Linguistik für den Ausbildungssektor, die helfen soll, gesellschaftliche Hürden für den Aufstieg niederzureißen. Oder wie es Utz Maas ausdrückt: es geht um den „bewusstgewordenen Skandal einer Linguistik, die blind für die gesellschaftlichen Aufgaben ist und doch dem gesellschaftlichen Aufgabenbereich ,Erziehungssektor" aufgezwungen wird“" (S. 8).

Auf den ersten Blick mögen also den Hintergrund die Bemühungen Anfang der 1970er Jahre bilden, die Linguistik in den Schulunterricht und damit in die Lehrerausbildung zu integrieren. Das Buch geht in seiner Darstellung über diesen Punkt jedoch deutlich hinaus. Letztlich geht es in der historischen Darstellung der Geschichte der Linguistik auch um die Frage, was der Gegenstandsbereich der Linguistik umfassen soll: Sollen Sprachen primär auf strukturellen Ebenen formal analysiert werden, so dass insbesondere die Rolle der sprachlich Handelnden ausgeblendet wird? Oder soll sich die Linguistik auch (oder gerade?) mit der Frage auseinandersetzen, was „Sprache als Bedingung und Leistung des gesellschaftlichen Handelns“ (S.13) ausmacht sowie der Frage nachgehen, was „die Entwicklung der Linguistik zur Entwicklung der Bedingungen der gesellschaftlichen Produktion und Reproduktion bei[trägt]“ (S. 290)? Zu dieser Perspektive auf Sprache kann die Generative Grammatik keine sinnvollen Beiträge leisten; gleichwohl ist sie das vorherrschende Paradigma der 1970er Jahre. Warum diese formalen Theorien des Strukturaufbaus so dominant geworden sind, ist somit das eigentliche Thema des geschichtlichen Abrisses. 
Das Buch kann man als aus drei Teilen bestehend auffassen, wobei der erste Teil aus der lesenswerten Vorbemerkung sowie den beiden ersten Kapiteln besteht (Linguistik und Bildungsreform; Sprachtheorie und Theorie der Gesellschaft). Dieser erste Teil beschäftigt sich mit den obigen Aspekten. Den weitaus größten Teil nehmen die Kapitel 3 bis 13 als zweiter Teil des Buchs ein, in denen die Entwicklung der Linguistik von Beginn des 20. Jahrhunderts bis zur Gegenwart der 1970er Jahre in Form der Generativen Transformationsgrammatik erläutert wird.

Inhaltlich überschneidet sich der zweite Teil des Buchs partiell mit anderen Werken zur Entwicklung der Linguistik wie z. B. Newmeyer (1986) zu den Ursprüngen und der Entwicklung der Transformationsgrammatik (in den USA) oder Seuren (1998) zur Geschichte der Linguistik von der Antike bis zur Gegenwart. Im Gegensatz zu diesen beiden Werken ist der Ausgangspunkt für Utz Maas' Werk die Beschäftigung mit der strukturalistischen Linguistik Bloomfieldscher Prägung, die er zu Beginn als Fortführung der Bestrebungen, die Sprachwissenschaft „wissenschaftlicher“ $\mathrm{zu}$ machen, erläutert. Im weiteren Verlauf des historischen Abrisses zeigt Utz Maas dann u.a., dass die Unterordnung der Linguistik unter die Psychologie seit den Junggrammatikern als selbstverständlich angesehen war, sowie die Verwurzelung der Generativen Grammatik im Strukturalismus. Vor allem weist Utz Maas darauf hin, dass bereits vor Chomsky andere Linguisten Fragen der Formalisierung adressiert haben. Die Aussage, Chomsky hätte diesbezüglich den entscheidenden Beitrag geleistet, ist falsch - eine Aussage, die sogar heutzutage im deutschen Wikipedia-Eintrag zu Noam Chomsky zu finden ist (,Noam Chomsky hat die Darstellung natürlicher Sprachen formalisiert“; letzter Zugriff: 13. 07. 2018). Utz Maas zeigt, dass algebraische Methoden schon deutlich länger bei der linguistischen Analyse verwendet wurden, und er zeigt vor allem detailliert, welchen gewichtigen Beitrag der akademische Lehrer Chomskys, Zellig Harris, für die Generative Grammatik geleistet hat.

Der dritte Teil ist das letzte Kapitel Warum ist die Linguistik, was sie ist? Dies ist eine Frage, die in der Linguistik in unregelmäßigen Abständen immer wieder aufgeworfen wird, wenn mittels neuer Methoden und Einsichten aus Nachbardisziplinen Gegenstandsbereiche der Linguistik neu justiert werden. Utz Maas' zentrale Aussage ist, dass die Linguistik bei der Theoriebildung von den Junggrammatikern bis zur Generativen Grammatik aufgrund der Formalisierungsbestrebungen einen Fortschritt verbuchen kann, während gleichzeitig die Aufgabenbestimmung der Linguistik durch ihre Einengung auf die Entwicklung formaler Regelsysteme rückschrittlich zu bewerten sei.

Warum lohnt es sich auch noch heute, sich mit dem Buch zu beschäftigen? Die aktuelle Lektüre dieses Buchs - 45 Jahre nach dem Erscheinen - lässt das 
Buch im Mittelteil zeitlos und im ersten sowie letzten Teil inhaltlich veraltet, aber auch merkwürdig modern erscheinen. Veraltet ist das Buch in den Teilen, die die gesellschaftliche und bildungspolitische Situation in der Bundesrepublik aufgreifen. Die bildungspolitischen Diskussionen werden in der im Buch angegebenen Form nicht mehr geführt, schon allein weil das Buch in der Beschreibung des Finalpunkts der Entwicklung der Linguistik eine Situation darlegt, die die Linguistik in Deutschland schon länger überwunden hat. Der Komplex „Linguistik und Schule“ ist selbstverständlich in der Lehrerausbildung präsent - man denke an die Lehramtsinitiative der DGfS - aber Utz Maas verstand den Beitrag der Linguistik für den Bildungssektor umfassender und radikaler als die Überführung linguistischer Inhalte in den Sprachunterricht.

Die Klassifikation von Formalisierungsgrad/Aufgabenbestimmung vs. Fortschritt/Rückschritt ignoriert die Tatsache, dass auch eine Soziolinguistik auf formale Strukturanalysen zurückgreifen muss. Sicherlich wird hierfür aber eine andere Art von Formalisierung benötigt als die, die die Generative Grammatik bietet.

Das Buch ist in seiner Ausrichtung auf „Sprache als Bedingung und Leistung der gesellschaftlichen Arbeit“ (S.7 in der 3. Auflage) hochaktuell in der heutigen Zeit, die sich durch sich gegenseitig bedingende politische, gesellschaftliche und technische Umbrüche auszeichnet. Die damalige Vorstellung von Linguistik in der Schule war, dass im Sinne der Bernsteinschen Soziolinguistik ein linguistisch orientierter Sprachunterricht „einen Beitrag zum Ausgleich sprachlich vermittelter Ungleichheit [...] leisten“ soll (S. 24). Zudem gilt, dass „[...] berufliche Ausbildung nie abgeschlossen, daß Weiterbildung für jeden zwingend ist“ (S.25). Beide Zitate stammen aus bildungspolitischen Dokumenten, die Ende der 1960er/Anfang der 1970er Jahre erschienen sind und in dem Buch zitiert werden. Wer politische Talkshows sieht, kennt insbesondere die letzte Aussage als eine Art Dauerschleife, über die Jahre hinweg produziert von Politikern jeder Couleur. Schaut man sich das Elend der heutigen Bildungspolitik an, stellt sich durchaus die Frage, wieso diese Einsicht in die Notwendigkeit des lebenslangen Lernens so alt und die Bildungserfolge schlechter geworden sind.

An die Stelle vermuteter sprachlich vermittelter Ungleichheit sind als reales Problem die vielfältigen kommunikativen Erscheinungen in mehrsprachigen Schulklassen mit Konsequenzen für den Bildungserfolg zu nennen. Das Buch tangiert mit seiner politischen Kritik zudem heutige Formen der Kommunikation von Politik und die Rezeption politischer Entscheidungen im Zeitalter der sozialen Medien. Wenn z. B. ein Kurznachrichten-Messengerdienst, der von seinen Nutzern in eine Empörungsmaschine transformiert wird, genutzt wird, um Politik zu simulieren bzw. sich darüber im Hysteriemodus zu echauffieren, so 
ist diese neu entstandene Textform nicht nur strukturell und pragmatisch interessant, sondern ein Fall für eine linguistisch orientierte Aufklärung über Prinzipien politischer Kommunikation. In diesen Kontext kann man auch die verkrampften Versuche einordnen, die derzeitigen Migrationsbewegungen sprachlich in den jeweiligen ideologisch geprägten Referenzrahmen zu pressen.

Es spricht also durchaus etwas für eine stärkere Berücksichtigung der sozialen und politischen Aspekte des sprachlichen Handelns in der Linguistik. Die Formalisierung muss dem nicht zwangsläufig im Wege stehen. Auch dies macht die Lektüre des Buchs von Utz Maas sichtbar.

\section{Literatur}

Ballmer, Thomas. 1985. Berliner Linguisten 1967-1982. In Thomas Ballmer \& Roland Posner (eds.), Nach-Chomskysche Linguistik, 36-52. Berlin: de Gruyter.

Newmeyer, Frederick. 1986. Linguistic theory in America. 2nd edn. Orlando: Academic Press. Seuren, Pieter. 1998. Western linguistics. An historical introduction. Oxford: Oxford University Press. 